\title{
Evaluación de la calidad del aire interior y exterior en un jardín de niños de la Ciudad de México
}

\author{
Marlene Cortez-Lugo, Ing. Amb., ${ }^{(1)}$ A driana Mercado-García, M.C., M.S.P., ${ }^{(2)}$ \\ Mauricio Hernández-Avila, M.C., D r. Sc., ${ }^{(2)}$ Fernando Meneses-González, M.C., M. Sc., (2) \\ Eduardo Palazuelos-Rendón, M.C. (3) $^{(3)}$
}

\begin{abstract}
Cortez-Lugo M, Mercado-García A, Hernández-Avila M, Meneses-González F, Palazuelos-Rendón E. Evaluación de la calidad del aire interior y exterior en un jardín de niños de la Ciudad de México.

Salud Publica Mex 1998;40:415-420.
\end{abstract}

\section{Resumen}

Objetivo. Evaluar las diferencias en los niveles de ozono $\left(\mathrm{O}_{3}\right)$ y bióxido de nitrógeno $\left(\mathrm{N} \mathrm{O}_{2}\right)$ en el interior y el exterior de una escuela en el suroeste de la Ciudad de México. Material y métodos. Se midieron diariamente estos contaminantes dentro y fuera del salón, de enero a abril de 1990, mediante monitoreo manual. Resultados. El $\mathrm{N} \mathrm{O}_{2}$ adentro y afuera no sobrepasó la norma mexicana $(0.21$ ppm). El coeficiente de correlación entre las concentraciones máximas de $\mathrm{NO}_{2}$ de la estación de monitoreo local de la ciudad y el exterior del salón fue de $0.82(p<0.001)$. En relación con el $\mathrm{O}_{3}$, la concentración máxima fuera del salón fue de $0.29 \mathrm{ppm}$, y adentro, de $0.17 \mathrm{ppm}$ (se colocaron en promedio por debajo de $0.06 \mathrm{ppm}$ ); entre el interior y el exterior del salón hubo una correlación de 0.72 y se observó que, por cada 1.7 ppm en el exterior, hay 1.0 ppm en el interior $(p<0.05)$. Conclusiones Las mayores concentraciones de $\mathrm{O}_{3}$ en el exterior se presentaron entre las 11:00 y las 14:00 horas, por lo que se recomienda tener el descanso previamente a este horario.

Palabras clave: ozono; dióxido de nitrógeno; monitoreo del ambiente; exposición a riesgos ambientales
Cortez-Lugo M, Mercado-García A, Hernández-Avila M, Meneses-González F, Palazuelos-Rendón E.

Evaluation of indoor and outdoor air quality

of a nursery school

in Mexico City.

Salud Publica Mex 1998;40:415-420.

\begin{abstract}
A bstract
Objective. To evaluate the differences between indoor and outdoor ozone $\left(\mathrm{O}_{3}\right)$ and nitrogen dioxide $\left(\mathrm{NO}_{2}\right)$ levels at a school located in southwest Mexico C ity. Material and methods Indoor and outdoor $\mathrm{O}_{3}$ and $\mathrm{N} \mathrm{O}_{2}$ levels were measured daily between January and A pril 1990 by manual monitoring. Results. Indoor and outdoor concentrations of nitrogen dioxide did not surpass the Mexican standard $(0.21 \mathrm{ppm})$. The correlation coefficient between maximum $\mathrm{N}_{2}$ concentrations measured by the city's local monitoring station and those measured outside the classroom was 0.82 ( $p<0.001$ ). Regarding ozone, its maximum outdoor concentration was $0.29 \mathrm{ppm}$ and indoor concentrations were on average below $0.06 \mathrm{ppm}$ (maximum = $0.17 \mathrm{ppm}$ ). The indoor/outdoor correlation coefficient was 0.72 , and for every $1.7 \mathrm{ppm}$ outside, there was $1.0 \mathrm{ppm}$ inside $(p<0.05)$ Conclusions. Since the highest outdoors $\mathrm{O}_{3}$ concentrations were observed between 11:00 and 14:00 $\mathrm{hrs}$, it is recommendable to have recess before this time.
\end{abstract}

Key words: ozone; nitrogen dioxide; environmental monitoring; environmental exposure

(1) Centro de Investigación en Salud Poblacional (CISP), Instituto N acional de Salud Pública (IN SP), México;A merican British Cowdray Hospital, México.

(2) CISP, IN SP, México.

(3) Secretaría del Medio Ambiente, Departamento del Distrito Federal, México.

Fecha de recibido: 7 de noviembre de 1997 - Fecha de aprobado: 20 de mayo de 1998

Solicitud de sobretiros: Ing. Marlene Cortez Lugo. Artificios 32, colonia Las Américas, Deleg. Alvaro 0 bregón, 01120 México, D.F., México. 
os problemas derivados de la contaminación del aire, agua, suelo y alimentos son ampliamente conocidos en la actualidad. Una gran parte de esos problemas son el resultado del rápido proceso de desarrollo e industrialización propio de las grandes ciudades, no obstante que se han puesto en marcha programas paralelos de protección al ambiente y a la salud de las poblaciones. ${ }^{1}$

El comportamiento fisicoquímico de los contaminantes desempeña un papel crucial en la contaminación atmosférica; las sustancias emitidas se dispersan en el aire, donde pueden interactuar químicamente con los componentes naturales de la atmósfera y con los materiales de la superficie.

La Ciudad de México, con 22 millones de residentes, está localizada a $2240 \mathrm{msnm}$, de tal manera que su atmósfera sólo contiene $77 \%$ de oxígeno, en comparación con el que existe en las localidades situadas al nivel del mar.

Por otra parte, está rodeada por montañas de más de $3000 \mathrm{~m}$ de altura, lo que limita la circulación del viento en un nivel superficial e incrementa la concentración de contaminantes; a ello se aúna la existencia de un clima subtropical, con una estación de lluvias de mayo a octubre y secas durante el resto del año. La altitud de la Ciudad de México contribuye a una ineficiente combustión de los vehículos de motor, lo que aumenta las emisiones de precursores de ozono.

Como resultado de las condiciones prevalecientes, en la Ciudad de México los niveles de distintos contaminantes han ido en aumento. Así, en el caso del ozono $\left(\mathrm{O}_{3}\right)$ las concentraciones máximas han rebasado la norma ( 0.11 ppm por hora) en más de $300 \%$ y se han mantenido por arriba de ésta, durante varias horas al día, en los últimos cinco años., ${ }^{2,3}$

Se han realizado evaluaciones de impacto en la salud de residentes de la Ciudad de México ${ }^{4,5}$ por la exposición al ambiente exterior con diversos niveles de ozono.

Esta investigación tiene como objetivo evaluar las diferencias en las concentraciones de $\mathrm{O}_{3}$ y bióxido de nitrógeno $\left(\mathrm{NO}_{2}\right)$ en interiores y exteriores de una escuela localizada en la zona suroeste de la Ciudad de México a través de una medición microambiental.

Una de las limitantes en la generación de modelos de exposición ambiental, en particular de $\mathrm{O}_{3^{\prime}}$ es la medición de exposición intramuros y extramuros.

La evaluación de las diferentes concentraciones de $\mathrm{O}_{3}$ y sus precursores, tanto en interiores como en exteriores, se ha documentado en otras ciudades, no así para la Zona Metropolitana de la Ciudad de México.

\section{Material y métodos}

Esta investigación se llevó a cabo en una escuela ubicada en la zona suroeste de la Ciudad de México, y forma parte de un estudio para evaluar los efectos sobre la salud por la exposición a ozono. ${ }^{4}$ Se realizaron mediciones diarias de $\mathrm{O}_{3}$ tanto en el interior como en el exterior del salón de clases de un jardín de niños, de enero a abril de 1990, de lunes a viernes exceptuando los días festivos.

El periodo de muestreo fue de cinco horas, dentro del horario de clases. El análisis se complementó con el procesamiento de datos de la Red Automática de Monitoreo Atmosférico (RAMA), Estación Pedregal, que es la más cercana a la escuela.

La determinación de las concentraciones de $\mathrm{O}_{3}$ en el interior y el exterior del salón de clases se realizó con el método de yoduro de potasio neutro, cuyo principio es la absorción del ozono en una solución de yoduro de potasio neutro para su posterior análisis espectrofotométrico. ${ }^{6}$

La medición de bióxido de nitrógeno en el interior y el exterior del salón de clases se realizó conforme al método modificado de Christie, ${ }^{7}$ que consiste en la absorción del bióxido de nitrógeno en una solución de arsenito de sodio/hidróxido de sodio, formando nitrito de sodio; la concentración de nitrito se mide colorimétricamente, previa adición de una solución de sulfanilamida/ácido fosfórico y clorhidrato de Tn-(1naftil)-etildiamina.

Para comparar las mediciones del exterior, tanto de $\mathrm{O}_{3}$ como de $\mathrm{NO}_{2^{\prime}}$, se obtuvieron las concentraciones de estos contaminantes de la Estación Pedregal de la RAMA; los analizadores que se utilizaron fueron: equipo Beckman, modelo 952A NO/ $\mathrm{NO}_{2} / \mathrm{NOx}$ Analyzer y equipo Thermo Electron Co., modelo 49 U.V. Photometric $\mathrm{O}_{3}$ Analyzer. El analizador de $\mathrm{NO}_{2}$ utiliza el método de detección de quimioluminiscencia, y el analizador de $\mathrm{O}_{3}$ utiliza el método de fotometría de luz ultravioleta.

Las concentraciones de $\mathrm{NO}_{2}$ y $\mathrm{O}_{3}$, determinadas por métodos analíticos, fueron previamente normalizadas con los analizadores automáticos respectivos, a fin de establecer una función que describiera los resultados del método analítico en relación con las lecturas del equipo automático.

El paso inicial consistió en la ejecución de muestreos simultáneos, induciendo una muestra de aire ambiente, tomada en un punto común hacia ambos sistemas (analítico y automático), garantizando así que tuviera la misma composición.

Para el caso del $\mathrm{O}_{3^{\prime}}$ estas muestras se tomaron durante periodos de 10 minutos hasta contar con, al 
menos, cinco mediciones para garantizar la validez estadística de la comparación. En el caso del $\mathrm{NO}_{2}$ los periodos de muestreo fueron de una hora. Las muestras obtenidas por los métodos manuales se analizaron mediante las técnicas analíticas correspondientes, y las concentraciones resultantes se relacionaron con las concentraciones promedio de las lecturas que registraron los analizadores automáticos durante los periodos respectivos.

Los resultados de los muestreos entre los analizadores automáticos y los métodos manuales fueron sometidos a un análisis de regresión lineal simple, donde: la variable dependiente fue la concentración determinada con el método manual (CM); la variable independiente, la concentración obtenida con el analizador automático (CA); el $\alpha$, el intercepto, y el coeficiente $\beta$, la unidad de cambio en la variable dependiente por cada cambio unitario en la variable independiente. Así, se obtuvo la siguiente ecuación:

$$
\mathrm{CM}=\alpha+\beta \mathrm{CA}
$$

Se compararon las concentraciones promedio horarias y las concentraciones máximas de ozono en 24 horas, así como las concentraciones promedio horarias de $\mathrm{NO}_{2}$ del interior y exterior del salón de clases, y entre el exterior del salón de clases y la RAMA Estación Pedregal. Asimismo, se hizo un análisis comparativo entre las mediciones máximas por día de $\mathrm{O}_{3}$ del interior y exterior de la escuela, y entre el exterior de la escuela y la Estación Pedregal.

De este modo, se observó el comportamiento diario de las concentraciones máximas de ambos contaminantes.

Se analizaron los datos de $\mathrm{O}_{3}$ y $\mathrm{NO}_{2}$ para obtener el coeficiente de correlación entre el interior y el exterior del salón de clases, y entre la estación de la RAMA y el exterior del salón de clases. Por último, se obtuvo un análisis de regresión lineal entre las concentraciones de $\mathrm{O}_{3}$ del interior y el exterior del sitio de estudio para establecer la asociación que existe entre ellos.

\section{Resultados}

\section{Bióxido de nitrógeno}

El intercepto y el coeficiente obtenidos mediante el análisis de regresión lineal simple, que se utilizaron para la normalización de los datos resultantes del método manual para el $\mathrm{NO}_{2^{\prime}}$ fueron: $\alpha=0.016$ y $\beta=0.59$; el modelo ajustó de manera adecuada $(r=0.84)$

Durante el periodo de estudio las concentraciones máximas de $\mathrm{NO}_{2}$ entre el interior y el exterior del salón de clases mostraron un comportamiento similar, aunque en el interior las concentraciones fueron menores; la medición máxima se registró entre las 11:00 y las 12:00 horas, con 0.125 ppm en el interior y 0.147 ppm en el exterior.

Las concentraciones máximas registradas en la estación de la RAMA y el exterior de la escuela rebasaron la norma entre las 9:00 y las 11:00 horas (0.148 ppm).

Las concentraciones promedio del comportamiento horario de $\mathrm{NO}_{2}$ aumentaron entre las 9:00 y las 10:00 horas, llegando a su pico máximo (0.07 ppm) entre las 10:00 y las 11:00 horas, para descender posteriormente. Las concentraciones promedio en el exterior son mayores que en el interior y mantuvieron el mismo comportamiento.

Se observó que las concentraciones promedio de $\mathrm{NO}_{2}$ registradas en la estación de la RAMA y el exterior de la escuela, tuvieron niveles similares. Las concentraciones promedio de $\mathrm{NO}_{2}$ obtenidas de la estación de la RAMA no rebasaron la norma $(0.21 \mathrm{ppm}$ promedio horario) (figura 1).

Se encontró un mejor coeficiente de correlación $(r=$ 0.82; $p<0.001$ ) entre los niveles máximos de $\mathrm{NO}_{2}$, de la estación de la RAMA y el exterior del salón de clases, en comparación con los niveles promedio $(r=0.42 ; p<$ $0.001)$; ambos fueron altamente significativos.

\section{Ozono}

El intercepto y el coeficiente obtenidos mediante el análisis de regresión lineal simple, que se utilizaron para la normalización de los datos resultantes del método manual para $\mathrm{O}_{3^{\prime}}$ fueron: $\alpha=0.02$ y $\beta=1.48$; si-

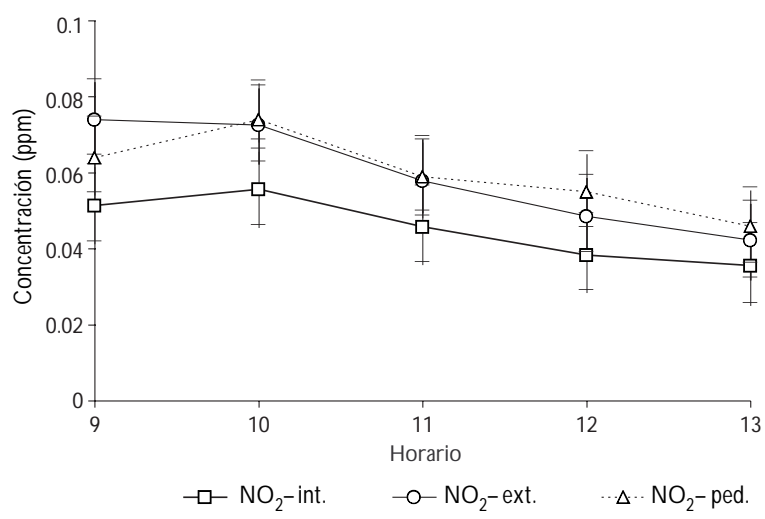

Figura 1. Bióxido de Nitrógeno: CONCENTRACIONES PROMEDIO HORARIAS EN EL INTERIOR Y EXTERIOR DEL SALÓN de clases y en la Estación Pedregal. México, eneroABRIL, 1990 
milarmente, el modelo ajustó de manera adecuada $(r=0.98)$.

Las concentraciones máximas de $\mathrm{O}_{3}$ sobrepasaron la norma (0.11 ppm promedio horario) durante el periodo de muestreo, tanto en el interior como en el exterior del lugar de estudio.

Se detectó una mayor concentración entre las 12:00 y las 14:00 horas, y se registró una concentración máxima de 0.17 ppm en el interior y de 0.29 ppm en el exterior. Las concentraciones fueron más altas en el exterior que en el interior a lo largo del periodo de estudio.

El comportamiento de las concentraciones promedio de $\mathrm{O}_{3}$ registradas en el exterior del salón de clases sobrepasaron la norma después de las 10:00 horas, y las cifras más altas se presentaron entre las 12:00 y las 13:00 horas; las concentraciones en el interior no rebasaron la norma.

El comportamiento entre las concentraciones promedio del interior y el exterior fue similar.

Las concentraciones promedio de $\mathrm{O}_{3}$ registradas en la Estación Pedregal y en el exterior del lugar de estudio tuvieron una correlación muy alta; los niveles sobrepasaron la norma entre las 11:00 y las 14:00 horas. Las concentraciones máximas diarias en el exterior del salón de clases y la Estación Pedregal mantuvieron niveles muy similares durante todo el periodo de estudio (figura 2).

$\mathrm{Al}$ comparar los niveles máximos de $\mathrm{O}_{3}$ y de $\mathrm{NO}_{2}$ se observa que el primero tiende a aumentar a medida que el segundo disminuye, debido a que el $\mathrm{NO}_{2}$ es un precursor del $\mathrm{O}_{3}$ (figura 3).

El coeficiente de correlación entre las mediciones máximas de $\mathrm{O}_{3}$ en el exterior del salón de clases y la

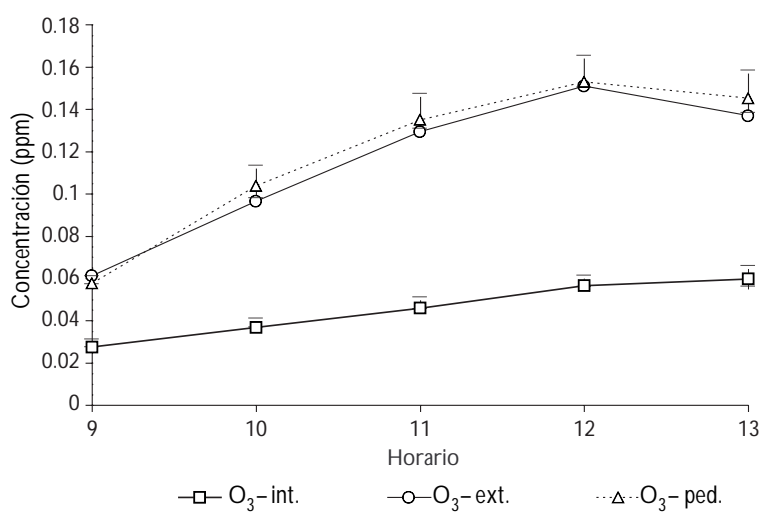

Figura 2. Ozono: concentraciones promedio hoRARIAS EN EL INTERIOR Y EXTERIOR DEL SALÓN DE CLASES y en la Estación Pedregal. México, enero-abril, 1990
Estación Pedregal fue altamente significativo $(r=0.97$, $p<0.001)$, al igual que la correlación entre las mediciones promedio de $\mathrm{O}_{3}$ del exterior del salón de clases y la Estación Pedregal $(r=0.99, p<0.001)$.

La correlación entre las concentraciones de $\mathrm{O}_{3}$ en el interior y el exterior del salón de clases fue significativa $(r=0.72, p<0.001)$ con una $\beta$ de 0.043 , mostrando una ligera sobrestimación de las mediciones en el exterior del salón de clases. Se observó una dispersión de los datos en la medida en que los niveles de $\mathrm{O}_{3}$ eran mayores (figura 4).

$\mathrm{Al}$ analizar las concentraciones de $\mathrm{O}_{3}$ máximas, mínimas y promedio horario en el interior del salón

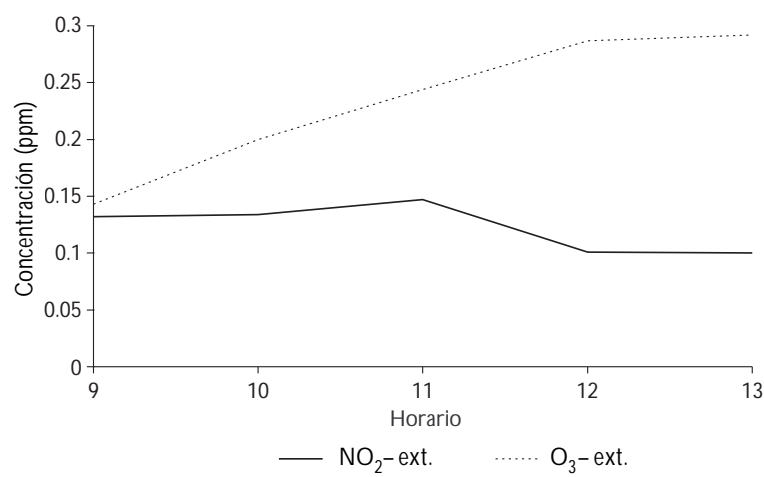

Figura 3. Concentraciones máximas de ozono y BIÓXIDO DE NITRÓGENO EN EL EXTERIOR DEL SALÓN DE CLASES. MÉxico, ENERO-ABRIL, 1990

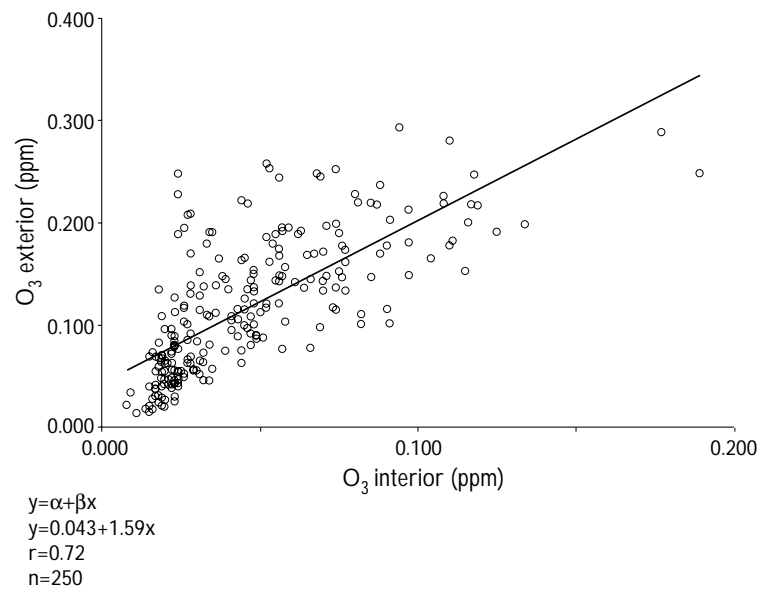

Figura 4. Comportamiento del ozono en el interior Y EXTERIOR del SALón de CLASEs. MéXico, enero-ABRIL, 1990

salud pública de méxico / vol.40, no.5, septiembre-octubre de 1998 
de clases, se observó que los niveles promedio se mantuvieron por debajo de la norma durante el horario de estudio, y sólo la rebasaron los niveles máximos entre las 12:00 y las 14:00 horas.

En el exterior se observó un paulatino incremento de los niveles de $\mathrm{O}_{3}$ durante el horario de estudio, que rebasó la norma entre las 11:00 y las 14:00 horas, mientras que las concentraciones máximas se mantuvieron por arriba de la misma.

La razón interior/exterior del $\mathrm{O}_{3}$ fue mayor entre las 9:00 y las 10:00 horas y disminuyó progresivamente (figura 5).

En la regresión lineal entre el interior y el exterior se observó que existe una asociación entre ambas mediciones; es decir, por cada 1.7 ppm en el exterior hay $1.0 \mathrm{ppm}$ en el interior, lo cual es estadísticamente significativo.

\section{Discusión}

A pesar de que ha transcurrido bastante tiempo desde que se llevó a cabo esta investigación, cabe destacar que el comportamiento del $\mathrm{O}_{3}$ y del $\mathrm{NO}_{2}$ notificado ha sido similar durante los últimos años, de tal manera que los resultados encontrados en este estudio no distan mucho de lo que se ha encontrado en trabajos más recientes. Por otro lado, existen pocos estudios realizados en escuelas que demuestren la relación interior/ exterior de las concentraciones de $\mathrm{O}_{3}$.

$\mathrm{El}$ resultado de la razón interior/exterior del $\mathrm{O}_{3}$ indica que los niveles son más bajos en el interior que en el exterior del salón de clases, por lo que es recomendable permanecer en el interior el mayor tiempo posible, de modo que se reduzca el riesgo de exponerse a altas concentraciones del contaminante.

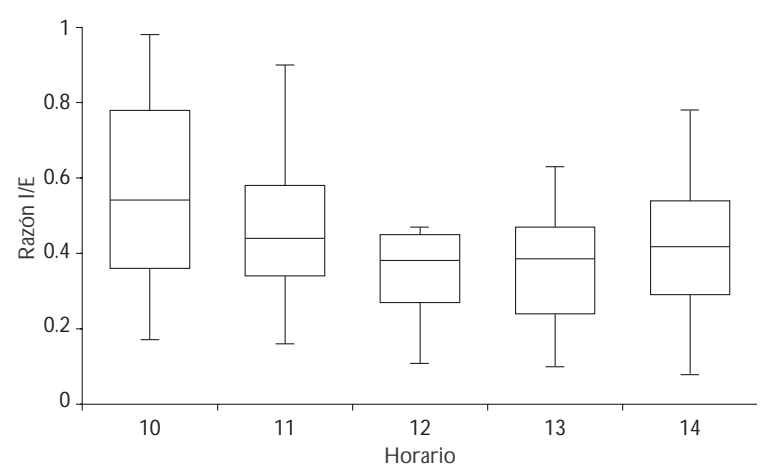

Figura 5. Comportamiento de la razón interior/ exterior del ozono en El SALón de CLASES. MéXICO, ENERO-ABRIL, 1990

salud pública de méxico / vol.40, no.5, septiembre-octubre de 1998
Como se mencionó ya, las concentraciones más altas de $\mathrm{O}_{3}$ en el exterior durante el periodo de estudio se presentaron entre las 11:00 y las 14:00 horas, excediendo la norma. Esa condición representa un riesgo para la salud de la población expuesta, ya que durante ese intervalo se ubica el receso de los prescolares, por lo que sería recomendable que el descanso fuera previo a este horario.

Se observó que en los muestreos realizados en el interior del salón de clases, cuando la puerta o las ventanas permanecían abiertas, las concentraciones de ozono se elevaban. Por lo tanto, es conveniente que las ventanas y puertas se mantengan cerradas al menos durante las horas en que las concentraciones de $\mathrm{O}_{3}$ están por arriba de la norma.

Las concentraciones de $\mathrm{O}_{3}$ en interiores pueden haberse modificado por la presencia de elementos a los cuales dicho contaminante es sumamente reactivo, como materiales plásticos, metales y telas, de tal manera que se han obtenido datos más reproducibles usando colectores de muestreos fabricados exclusivamente con teflón o con vidrio. ${ }^{8}$

Para reducir los efectos nocivos a la salud asociados con las altas concentraciones de $\mathrm{O}_{3^{\prime}}$ debe evitarse en lo posible salir a exteriores cuando se presentan situaciones de contingencia. Por lo anterior, es importante evitar hacer ejercicio al aire libre durante las horas en que dichas concentraciones son mayores.

Es conveniente realizar estudios para evaluar tiempos más prolongados de exposición a estos contaminantes del aire intra y extramuros y realizar mediciones que indiquen una relación más certera entre las concentraciones de los contaminantes y sus posibles efectos sobre la salud.

Los estudios epidemiológicos enfocados a la depuración ambiental por contaminantes del aire son sumamente importantes como indicadores de riesgo para las poblaciones sujetas a la contaminación, así como auxiliares para prevenir su exposición y consecuencias pero, sobre todo, para establecer las normas de calidad de aire que correspondan a las características propias de cada país. Lo anterior facilitaría la planeación y la toma de decisiones para el diseño de estrategias y actividades dirigidas a proteger la salud y a controlar las fuentes de contaminación.

\section{Referencias}

1. Romieu I,W eitzenfeld $\mathrm{H}$, Finkelman J.U rban air pollution in Latin A merica and the Caribbean. J Air W aste Manage Assoc 1991;41:1166-1171. 2. Secretaría de D esarrollo U rbano y Ecología. La contaminación atmosférica en la Ciudad de México. Boletín Informativo del DDF 1988. 
3. Secretaría de Desarrollo Urbano y Ecología. Reporte anual sobre la calidad del aire en la Ciudad de México. Boletín Informativo del DDF 1991.

4. Romieu I, C ortez-Lugo M, Ruiz-Velasco S, Sánchez S, Meneses F, Hernández M. Air pollution and school absenteeism among children in Mexico City. Am J Epidemiol 1992;136(12):1524-1531.

5. Castillejos M, Gold D, Dockery D, Tosteson T, Baum T, Speizer PE. Effects of ambient ozono on respiratory function and symptoms in school children in Mexico City. Am Rev Resp D is 1992;145:276-282.

6. Método equivalente para la determinación de ozono en la atmósfera No. P\&CAM 154. Método de yoduro de potasio neutro. Métodos de laboratorio para la determinación de contaminantes atmósfericos. MéxiCO, D.F.: UAM-Azcapotzalco, 1984:87-94.

7. Método equivalente para la determinación de bióxido de nitrógeno en la atmósfera. EPA. Método del arsenito de sodio, modificación de la técnica de Chirstie. Métodos de laboratorio para la determinación de contaminantes atmosféricos. México, D.F.: UAM-Azcapotzalco, 1984:41-46. 8. O rganización Panamericana de la Salud, O rganización Mundial de la Salud. 0 xidantes Fotoquímicos. México, D.F.: O PS-O MS, Criterios de Salud Ambiental, núm. 7, 1980:1-83. 